\title{
Multidisciplinary multi-objective design optimization of an active morphing wing section
}

\author{
Florian Dexl $^{1} \cdot$ Andreas Hauffe $^{1} \cdot$ Klaus Wolf $^{1}$ \\ Received: 17 February 2020 / Revised: 8 April 2020 / Accepted: 16 April 2020 / Published online: 20 July 2020 \\ (C) The Author(s) 2020
}

\begin{abstract}
Design and optimization of morphing wings are of current research interest as they promise increasing efficiency and flexibility of future aircraft. A challenging task is to find structural layouts of morphing wings that enable aerodynamically optimized shape changes without defining the target shape a priori. The current paper addresses this task and presents a method that combines the optimization of the active structure of a wing section, parameterized by Lindenmayer cellular systems, with an aerodynamic evaluation. Neither the structural layout nor the target shape has to be defined a priori. This aim is achieved by a multidisciplinary optimization using evolutionary algorithms with aerodynamic and structural objectives. The developed method allows to optimize the topology of the internal structure, the placement of linear contraction, and expansion actuators as well as the setting of their actuation degree concurrently. It is shown that the present approach allows to find optimized internal layouts containing active structural elements for morphing wing sections.
\end{abstract}

Keywords Morphing wing section $\cdot$ Drag reduction $\cdot$ Topology $\cdot$ Evolutionary optimization $\cdot$ Fluid-structure interaction (FSI) - Lindenmayer cellular systems

\section{Introduction}

According to the Flightpath 2050 of the European Commission (2011), $\mathrm{CO}_{2}$ emissions of commercial aircraft shall be reduced by $75 \%$ in the year 2050 , compared with the state of the art in 2000. This require defines a challenging task for future aircraft developments and creates the need of game-changing technologies. Morphing wings are a promising possible solution to improve the aerodynamic efficiency.

The aerodynamic performance of conventional aircraft wings is optimized for their design regime, which is mainly

Responsible Editor: Joaquim R. R. A. Martins

Florian Dexl

florian.dexl@tu-dresden.de

Andreas Hauffe

andreas.hauffe@tu-dresden.de

Klaus Wolf

klaus.wolf@tu-dresden.de

1 Institute of Aerospace Engineering, Technische Universität Dresden, 01062 Dresden, Germany cruise flight. Therefore, their efficiency is often reduced under other flight conditions. Flaps and slats offer only limited adaptive capabilities. Morphing wings promise to overcome these non-optimal solutions by adapting the wing geometry to an aerodynamically optimal shape for every flight condition. Comprehensive reviews of morphing wing technologies and suitable analysis methods have been published by Barbarino et al. (2011) and Li et al. (2018). Generally, morphing wings are classified based on their morphing mode in planform morphing, out-of-plane morphing, and airfoil morphing. The current work addresses airfoil morphing by considering the cross section of an infinite wing.

As stated by Vasista et al. (2012), the design of morphing wings is a highly multi-objective and multidisciplinary task. In general, both the optimal geometrical change of the wing section contour and the structural design enabling this shape change are unknown a priori. As the structure of a morphing wing section directly affects the achievable shape changes and thereby the possible aerodynamic benefits, new concurrent optimization approaches for the internal structure and the resulting morphed shape are required. Additionally, for active morphing wings, actuators must be taken into account. Figure 1 outlines the resulting multidisciplinary aspects of design and optimization. 


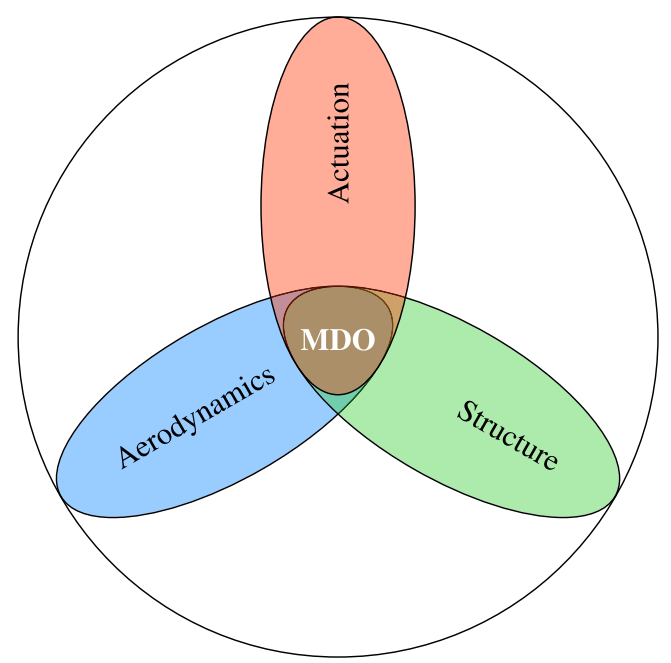

MDO: Multidisciplinary Design and Optimization

Fig. 1 Multidisciplinary aspects of the design and optimization of active morphing wings

In the following, a brief literature review on existing optimization methods for morphing airfoils and wing sections is given.

Aerodynamic optimization: Secanell et al. (2006) used aerodynamic shape optimization for generating airfoils optimized for different flight phases and analyzed the results regarding possible morphing mechanisms. Further, the reduction of the required actuation energy for shape deformations was taken into account in an optimization of morphing airfoils by Namgoong et al. (2012). An aerodynamic optimization with restrictions to morphing modes provided by the Fishbone Active Camber (FishBAC) mechanism was performed by Fincham and Friswell (2015). Fusi et al. (2018) optimized morphing airfoils for helicopter rotor blades.

Structural optimization: Additionally, research has been done on optimization methods for designing active internal structures that enable the deformation into a predefined target shape. A compliant trailing edge flap with fixed actuators was optimized by Friswell et al. (2006). Baker and Friswell (2009) described the optimization of an active morphing wing section by placing linear actuators on a truss ground structure for achieving a predefined trailing edge deflection of a symmetric base profile. The optimization of an internal mechanism with the objective of achieving a predefined target deformation of the leading and trailing edge was done by De Gaspari and Ricci (2011).

Aerostructural optimization: For taking multidisciplinary aspects of morphing wing sections into account, aerostructural optimizations have to be performed. Strelec et al.
(2003) described the aerostructural optimization of a wing section with a NACA 0012 base profile with three added shape memory material (SMA) actuators. Genetic algorithms were used to find optimal solutions for the start and end points as well as the expansion of the SMA actuators in order to maximize the lift-to-drag ratio. Maute and Reich (2006) used the solid isotropic material with penalization (SIMP) approach for optimizing the design of a morphing mechanism with fixed actuator positions for minimizing an exemplary drag objective function. The optimization of helicopter rotor blades with the objective of varying the moment coefficient with mass reduced active internal structures was described by Seeger (2012). A concurrent optimization of a three-dimensional external wing shape, the internal compliant structure, and actuator positions of active ribs for spanwise airfoil morphing was performed by Molinari et al. (2014). Woods and Friswell (2016) used genetic algorithms for the optimization of the FishBAC concept reducing the aerodynamic drag, the structural mass, and the actuation energy in a multi-objective problem.

Aim of the presented method: A new modeling and optimization method in order to design and assess structural concepts for active morphing wing sections is presented. By optimizing the structural layout of the wing section and the morphed airfoil shapes concurrently, the multidisciplinary aspects are consequently taken into account.

As the main aim is to provide a general optimization method for active morphing wing sections, manufacture constraints are not considered. Due to this, specific actuator technologies and structural failure are also not taken into account. The present method allows to find new concepts for morphing wing sections that can be further developed towards practical realization.

Focus is set on active morphing wing section structures containing freely placed linear actuators to enable a holistic shape change over varying flight conditions, as depicted in Fig. 2. As a key feature, neither the structural layout nor the

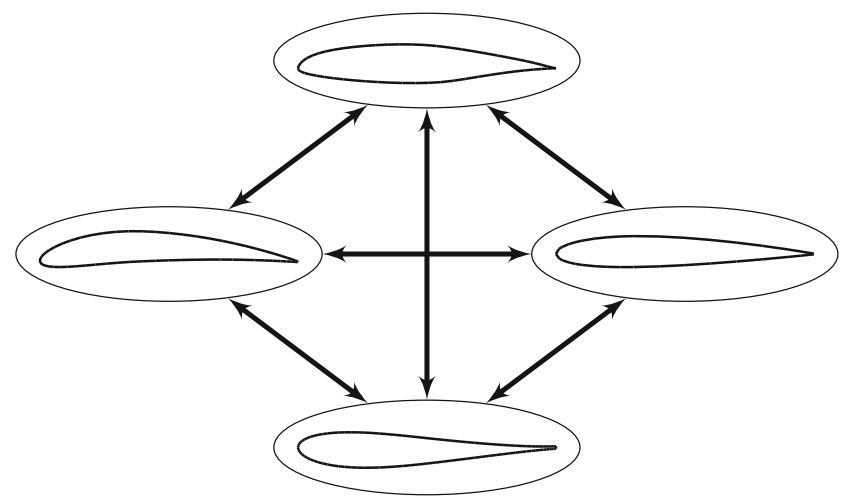

Fig. 2 Understanding airfoil morphing as the holistic change between freely deformed airfoils 
target shape has to be defined a priori. In addition, emphasis has been put on the definition of optimization objectives and constraints that lead to feasible deformation modes despite the largely unconstrained deformation possibilities of the actuated wing section.

\section{Problem formulation}

Conventional airfoils with a fixed geometry offer their best aerodynamic performance, interpreted here as a minimal drag coefficient $c_{d}$, only in a limited range of lift coefficients $c_{l}$, as exemplified for the NACA 2412 airfoil in Fig. 3.

The addressed task is to overcome this limitation by drag reduction for a wide range of flight conditions. Therefore, the target is to minimize the drag coefficient $c_{d}\left(c_{l}\right)$, as depicted by the arrows in Fig. 3. Constraints arise from the demand for aerodynamically robust and feasible airfoil shapes in every morphed state and a low structural mass. Therefore, four targets for morphing wing sections are considered here:

1. Low aerodynamic drag over a wide range of flight conditions,

2. Aerodynamically robust morphed airfoil shapes,

3. Aerodynamically feasible morphed airfoil shapes,

4. Low structural mass.

Firstly, a general concept of the active morphing wing section is developed which is shown in Fig. 4. The concept consists of a predefined outer contour with variable skin thickness and an internal structure consisting of passive members as well as active contraction and expansion members. Passive components are non-actuated structures; active components are interpreted as linear actuators. A

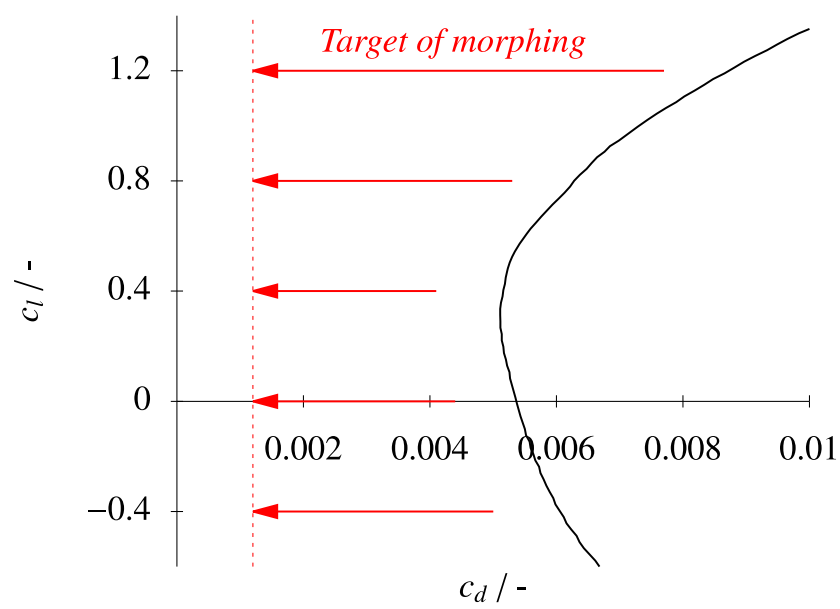

Fig. 3 Example of a drag polar for the NACA 2412 airfoil at a Reynolds number of $10^{7}$, calculated with XFOIL; arrows and dashed line indicate the changes intended by airfoil morphing fixed rectangular spar area is placed at $25 \%$ of the chord length $l$.

When defining specific morphing wing section structures based on this general concept, a large number of questions arise. The essential ones of them are listed in the following:

- Layout of the internal structure?

- Placement of actuators?

- Definition of actuation schemes?

- Definition of actuation strength?

- Stiffness distribution over the outer skin?

- Aerodynamic quality of the morphed airfoil shapes?

- Assessment of aerodynamic and structural objectives?

Due to the large number of variables and objectives, an optimal solution cannot be found intuitively. This makes the development of a sophisticated optimization framework necessary. To achieve this, no constraints on the internal structural layout are taken into account.

\section{Modeling framework}

\subsection{Aims and scope}

The developed framework shall offer as much flexibility as possible, in order to supply a well-founded basis for future adaptions. Therefore, focus has been put on selecting parameterization methods that allow a sufficient design space by a low number of design variables. For the aerodynamic and structural evaluation, methods of low order but high computational efficiency have been chosen. The modular approach of the developed framework allows to replace both the parameterization methods and the evaluation tools by more sophisticated methods in future developments.

\subsection{Parameterization}

For optimizing the described morphing wing section concept, the layout of the internal structure, the placement, and the actuation degree of the active members as well as the thickness of the wing section contour have to be parameterized efficiently.

\subsubsection{Structural layout}

For the layout of the inner structure, emphasis has been placed upon selecting a method that allows a flexible description of a structure consisting of linear members with few parameters. Infeasible designs, for example topologies with an open load path, shall be avoided. For that purpose, methods describing cellular structures have been chosen. 

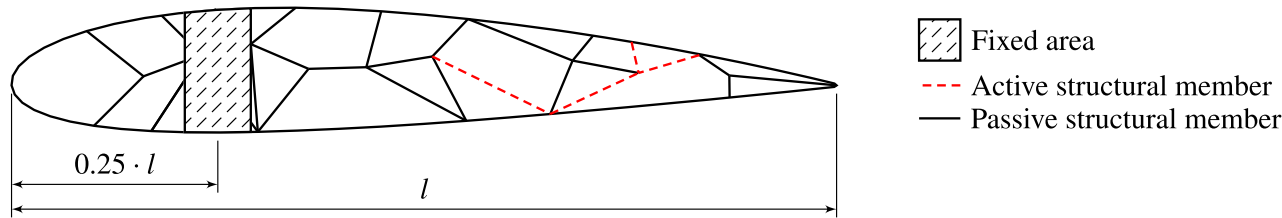

- Passive structural member

Fig. 4 General concept of the morphing wing section

Rather new parameterization methods are cellular division-based methods (Deaton and Grandhi 2014). As in Pedro and Kobayashi (2011), Lindenmayer cellular systems, namely mBPMOL systems, proposed by Prusinkiewicz and Lindenmayer (1990), are used here.

Lindenmayer cellular systems are defined by an alphabet $\Sigma$, an axiom $\omega$, a set of rules $P$, and a maximum number of division stages $n_{d i v}$. To parameterize the structural layout, the axiom $\omega$ and the set of rules $P$ are encoded by integers. For further details on the creation of Lindenmayer cellular systems, the reader is referred to Pedro and Kobayashi (2011). In contrast to Pedro and Kobayashi (2011), the osmotic pressure is neglected during the cellular division.

An arbitrary polygon can be used as the starting cell. As the number of variables defining the cellular system increases with the number of edges of the starting cell, an equilateral triangle is used as the starting cell. For demonstrating the present method in accordance with the aims described in Section 3.1, this choice minimizes the number of design variables and still offers a sufficiently large design space.

After the subdivision of the edges according to their production rules and placement of the markers, a cellular subdivision is performed, as shown in Fig. 5. Every closed region is searched for a matching pair of markers, pointing into the region. The next step is to check if the subdivision of



(a) Start with unit triangle

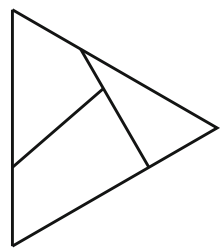

(c) $2^{\text {nd }}$ cellular subdivision

Fig. 5 Exemplary subdivision process of Lindenmayer cellular systems from the starting cell (a) to the first (b), second (c), and third (d) cellular subdivision the region at the connection line between the two matching markers leads to two feasible new regions. Regions are defined as feasible when their smallest interior angle is larger than a defined minimum interior angle $\varphi_{\min }$ and the area of the smaller resulting region is greater than a defined ratio $r_{A}=(0 \ldots 0.5)$ of the original area. Additionally, the edge lengths of the resulting cells are checked to be greater than a minimum length $l_{e, \min }$. If all three conditions are fulfilled, the region is divided and further markers in this area are ignored. This cellular subdivision process is repeated $n_{\text {div }}$ times.

The resulting cellular system is then mapped from the unit triangle onto the wing section contour by a common starting point and the normalized arc length coordinate $s$ along the contour in counterclockwise order, as shown in Fig. 6.

\subsubsection{Active linear elements}

Active areas are placed on the cellular structure and the outer wing section contour using moving morphable components (MMCs) proposed by Guo et al. (2014).

Every MMC is defined by a length $l$, a width $w$, two center coordinates $\left(x_{0}, y_{0}\right)$, and a rotation angle $\Theta$, as shown in Fig. 7. In the optimization, a variable number of $n_{M M C}$ MMCs are placed on the internal structure. Their number, shape, and position are varied during the optimization process.

The edges of the Lindenmayer cellular system are defined as active members, when their midpoint is enclosed by a MMC. Furthermore, the parts of the wing contour encompassed by a MMC are also defined as active members. Every MMC $j=1,2, \ldots, n_{M M C}$ is linked to a set of actuation degrees $\mathbf{T}_{j}=\left[T_{j, 2}, T_{j, 3}, \ldots, T_{j, n_{F C}}\right]$ for the different flight conditions $i=2,3, \ldots, n_{F C}$. For flight condition $i=1$, the airfoil is not actuated so the actuation degree is set to zero, i.e., $T_{j, 1}=0$. By this, the position of the actuators is constant for each flight condition $i$, but the actuation degrees are set individually.

\subsubsection{Structural thicknesses}

As the morphing wing section is designed with an integral outer contour without distinct joints, the structure acts as a compliant mechanism. Therefore, influence on the 
Fig. 6 Principle of mapping the Lindenmayer cellular system from the unit triangle to the wing section contour by a common starting point

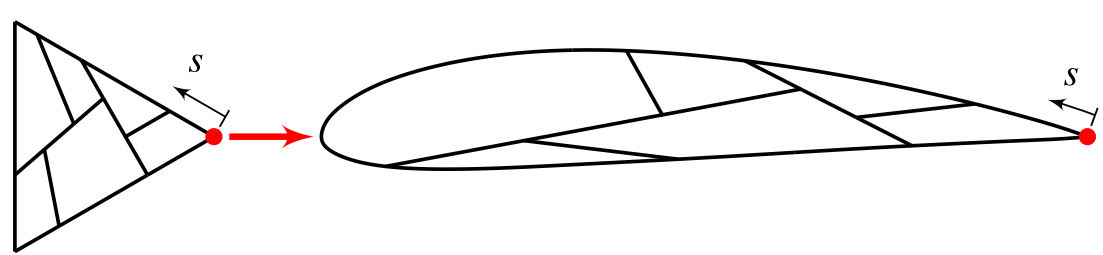

structural compliance of the wing skin has to be allowed in the optimization process. This is done by parameterizing the thickness of the wing skin. The thickness is described by twelve supporting points $t_{k}, k=1,2, \ldots, 12$ along the outer contour, as shown in Fig. 8. In between these points, the thickness is interpolated linearly. The thickness of the internal structure is constant and parameterized by the optimization variable $t_{\text {internal }}$.

\subsection{Aerostructural evaluation}

The aerostructural evaluation is performed using the fluidstructure interaction approach outlined in Fig. 9. In this approach, the in-house linear static finite element (FE) solver FiPPS ${ }^{2}$ is coupled with the open-source two-dimensional viscous-inviscid panel code XFOIL 6.99 (Drela 1989). Although XFOIL implements a low-fidelity aerodynamic solver, it offers good accuracy in calculating the aerodynamic characteristics of common airfoils, as the comprehensive comparison with Reynolds-averaged Navier-Stokes (RANS) methods by Herbert-Acero et al. (2015) has shown. The computational effort of XFOIL is magnitudes lower than those of RANS methods, what makes it well suited for the present optimization method. From the structural mechanics point of view, it is sufficient to substitute the three-dimensional wing segment by a two-dimensional framework, since no spanwise effects are considered in this research. Therefore, Euler-Bernoulli beam elements have been chosen to model the cross section of the wing structure. Data exchange between FiPPS ${ }^{2}$ and XFOIL is realized by input- and output-files.

During the fluid-structure interaction, XFOIL is used in inviscid mode for calculating the aerodynamic loads of the deformed wing section, which are applied to the undeformed FE model. This allows an efficient realization of the fluid-structure interaction, since the Cholesky
Fig. 7 Moving morphable components (MMCs) define areas of active linear elements on the base structure and the wing section contour

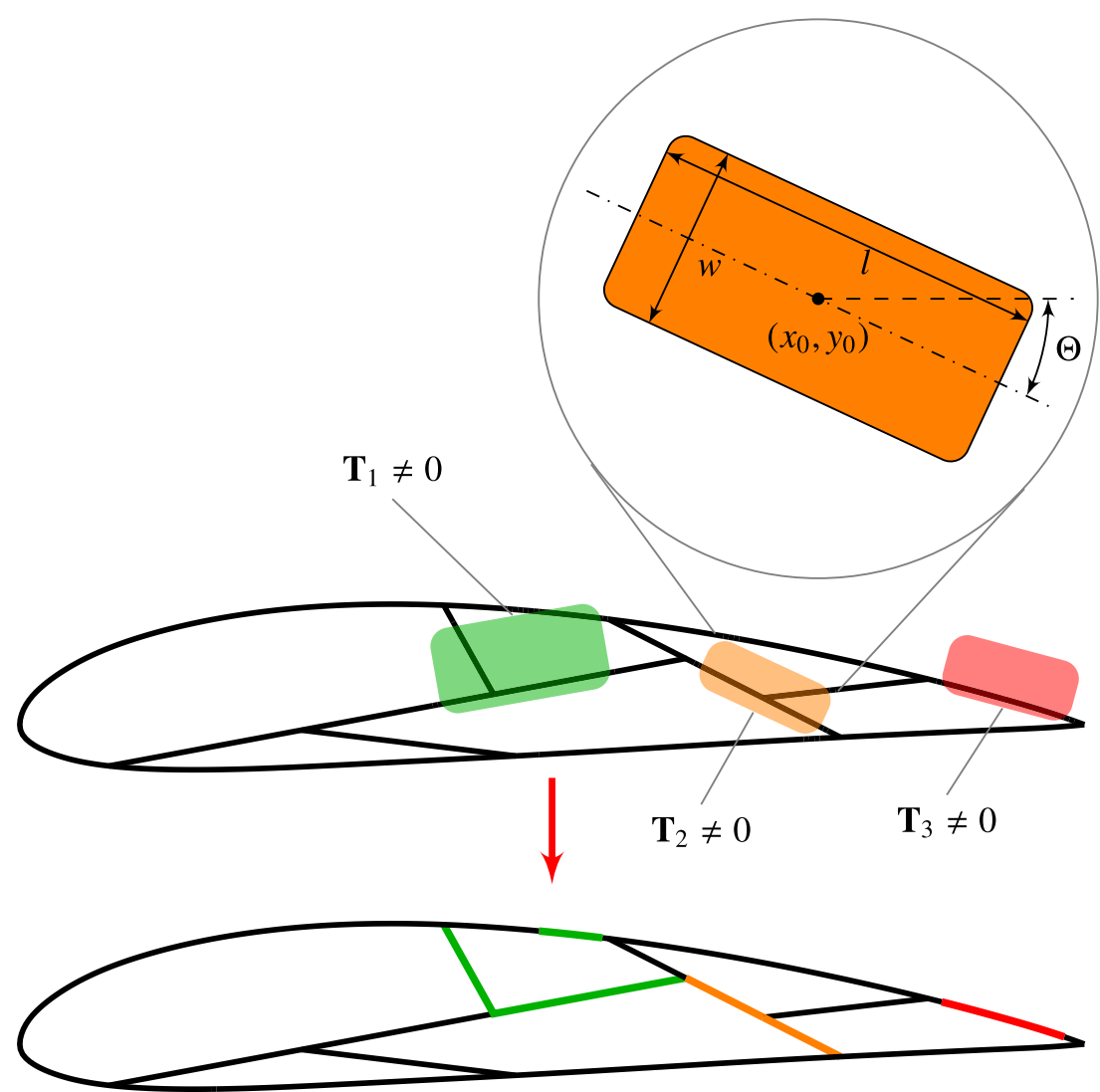


Fig. 8 Supporting points $t_{k}$ for defining the structural thickness of the wing section skin

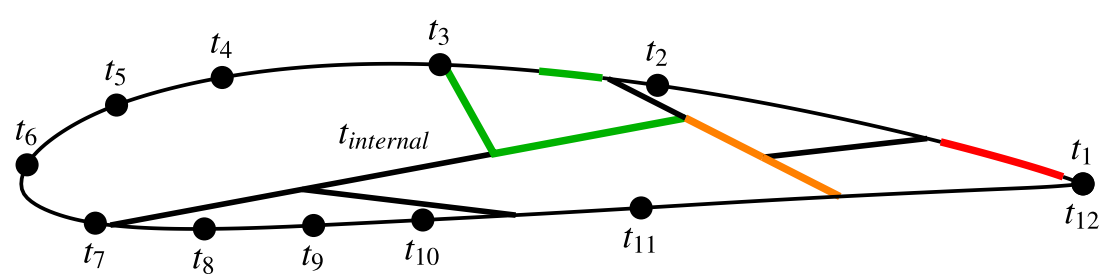

decomposition of the stiffness matrix is only performed once by the FE solver. This procedure is repeated until the stopping criterion $\varepsilon_{u}=1 \%$ (see Fig. 9) is reached. This criterion is based on the relative change of the maximum deformation over all FE nodes $n=1,2, \ldots, n_{\text {nodes }}$

$\delta u_{\text {max }}=\left|\frac{\max _{n} u_{n}^{k}-\max _{n} u_{n}^{k-1}}{\max _{n} u_{n}^{k}}\right|$

between two iteration steps $k$ and $k-1$. In terms of the aims described in Section 3.1, this deformation-based criterion has been chosen in order to obtain a good balance between computation time and accuracy.

After convergence of the fluid-structure interaction, the geometry deformed by actuation and aerodynamic loads is obtained.



Fig. 9 Outline of the fluid-structure interaction process

\subsection{Modeling of actuators}

Linear actuators are simulated by equivalent thermal loads $T_{j, i}$ that are applied to the respective structural elements. With the artificial thermal expansion coefficient $\alpha_{T}$, the corresponding actuator strain is:

$\varepsilon_{j, i}=\alpha_{T} T_{j, i}$.

An artificial thermal coefficient $\alpha_{T}$ of unity is set for the active components of the internal structure in order to model the expansion or contraction of the linear actuators. The remaining material properties are identical over the whole structure.

\subsection{Boundary conditions}

Displacement boundary conditions are chosen to model a fixed spar. The area from 20 to $30 \%$ of the chord length is fixed, as shown in Fig. 10. The internal structure in this area is cut at the intersection edges and the resulting free edge points are fixed at all translational and rotational degrees of freedom. In addition, the upper and lower side in the defined spar area is fixed.

\section{Optimization framework}

As stated in Section 2, multiple flight conditions $i=$ $1, \ldots, n_{F C}$ have to be taken into account for the optimization effort. Every flight condition (FC) is specified by a given lift coefficient $c_{l, i}$, a Mach number

$M a_{i}=\frac{u_{\infty}}{a_{i}}$

and a Reynolds number

$R e_{i}=\frac{\rho_{I S A}\left(H_{i}\right) u_{\infty} l}{\eta_{i}}$,

where $u_{\infty}$ is the flight speed, $a_{i}$ the speed of sound, $\rho_{I S A}\left(H_{i}\right)$ the air density according to the International Standard Atmosphere (ISA) for flight altitude $H_{i}$, and $\eta_{i}$ the dynamic viscosity of air. For the first flight condition $i=1$, the morphing wing section is unactuated and represents the base airfoil contour, solely deformed by the aerodynamic loads. For every other flight condition $2 \leq i \leq n_{F C}$, 
Fig. 10 Area of fixed degrees of freedom

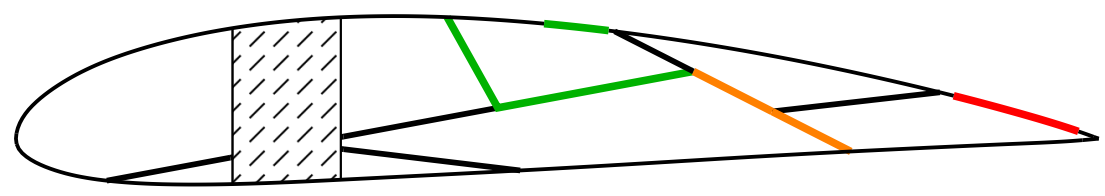

27 Fixed area

the wing section will be actively deformed to an optimized shape.

Therefore, the main target is to minimize the drag coefficient $c_{d}$ over all flight conditions $i$ by the optimized morphing wing section

$\min \left\{\max _{i}\left[c_{d}\left(c_{l, i}, M a_{i}, R e_{i}\right)\right]\right\}, i=1,2, \ldots, n_{F C}$,

as well as the structural mass $m$. As explained in Section 2, the aim for aerodynamically feasible morphed wing section shapes leads to additional targets that are explained in the following.

\subsection{Aerodynamic objectives}

It is a challenging task to find appropriate aerodynamic objectives for freely deforming airfoils. As outlined by Drela (1998), a single-point optimization of airfoils mainly results in infeasible contours. For the present optimization problem, a set of three aerodynamic objectives proved to be appropriate, which have been identified by a large number of purely aerodynamic airfoil optimizations.

The first of these objectives is to find the minimal drag coefficient for the given lift coefficient

$c_{d}=c_{d}\left(c_{l}\right)$

resulting in low-drag airfoils. Induced drag is not taken into account, as an infinite wing is considered.

Second, the standard deviation $\sigma_{d}$ of the drag coefficient resulting from small changes of the angle of attack by $\Delta \alpha=$ $\pm 1^{\circ}$ around $\alpha=\alpha\left(c_{l}\right)$ has to be minimized

$$
\begin{aligned}
\sigma_{d}= & {\left[\frac{c_{d, \alpha}^{2}+c_{d, \alpha-\Delta \alpha}^{2}+c_{d, \alpha+\Delta \alpha}^{2}}{3}-\right.} \\
& \left.\left(\frac{c_{d, \alpha}+c_{d, \alpha-\Delta \alpha}+c_{d, \alpha+\Delta \alpha}}{3}\right)^{2}\right]^{0.5},
\end{aligned}
$$$$
\text { with } c_{d, \alpha}=c_{d}(\alpha) \text {, }
$$

in order to obtain aerodynamically robust airfoils.
Furthermore, the kinematic shape factor $H_{K \max }$ of the boundary layer theory should be minimized

$$
H_{K \max }=\max _{s}\left[H_{K}(s)\right]=\max _{s}\left[\frac{\delta^{\star}(s)}{\Theta(s)}\right]
$$

where $\delta^{\star}$ is the displacement thickness, $\Theta$ the momentum thickness of the boundary layer, and $s$ the arc length coordinate of the upper and lower contour. This objective is important to obtain feasible airfoils without significant separation regions.

\subsection{Structural objectives}

The morphing wing section structure shall have a minimal mass. Therefore, in addition to the aerodynamic objectives, the specific mass $\mu$ of the structure has to be minimized. The specific mass is defined as the total structural mass $m$ per segment width $w_{a f}$ in spanwise direction:

$\mu=\frac{m}{w_{a f}}$.

It can be interpreted as the linear mass density of the wing segment. A minimal mass design is the only structural objective taken into account.

\subsection{Optimization constraints}

Optimization constraints penalize infeasible designs. If a constraint is violated during the optimization process, the objective values are multiplied with penalization parameters $w$ which are several orders of magnitude larger.

Infeasible deformations include intersections of the upper and lower half of the morphed wing contour as well as the case that the inner structure penetrates the outer contour under aerodynamic and actuation loads. Additionally, the area $A_{i}$ enclosed by the outer contour when actuated for flight condition $i$ may not change more than $10 \%$. For restricting the angle of attack variation between the flight 
Table 1 Number of integer and floating point optimization variables

\begin{tabular}{|c|c|c|}
\hline \multirow[t]{2}{*}{ Purpose } & \multicolumn{2}{|c|}{ Number of variables } \\
\hline & Integer & Floating point \\
\hline Axiom $\omega$ & 3 & - \\
\hline Set of rules $P$ & 36 & - \\
\hline Number of cellular divisions $n_{d i v}$ & 1 & - \\
\hline Minimal inner angle $\varphi_{\min }$ & - & 1 \\
\hline Minimal area ratio $r_{A}$ & - & 1 \\
\hline Minimal edge length $l_{e, \min }$ & - & 1 \\
\hline Number of MMCs $n_{M M C}$ & 1 & - \\
\hline Length of MMCs $l$ & - & $n_{M M C}$ \\
\hline Width of MMCs $w$ & - & $n_{M M C}$ \\
\hline Center of MMCs $\left(x_{0}, y_{0}\right)$ & - & $2 n_{M M C}$ \\
\hline Rotation angle of MMCs $\Theta$ & - & $n_{M M C}$ \\
\hline Actuation degrees $T_{j, i}$ & - & $\left(n_{F C}-1\right) n_{M M C}$ \\
\hline Contour thicknesses $t_{k}$ & - & 12 \\
\hline Internal thickness $t_{\text {internal }}$ & - & 1 \\
\hline Total & 41 & $16+\left(4+n_{F C}\right) n_{M M C}$ \\
\hline
\end{tabular}

conditions, their difference to flight condition 1 is limited to $2^{\circ}$. The rational behind this is to avoid exceeding variations of the angle of attack instead of shape adjustments by active morphing.

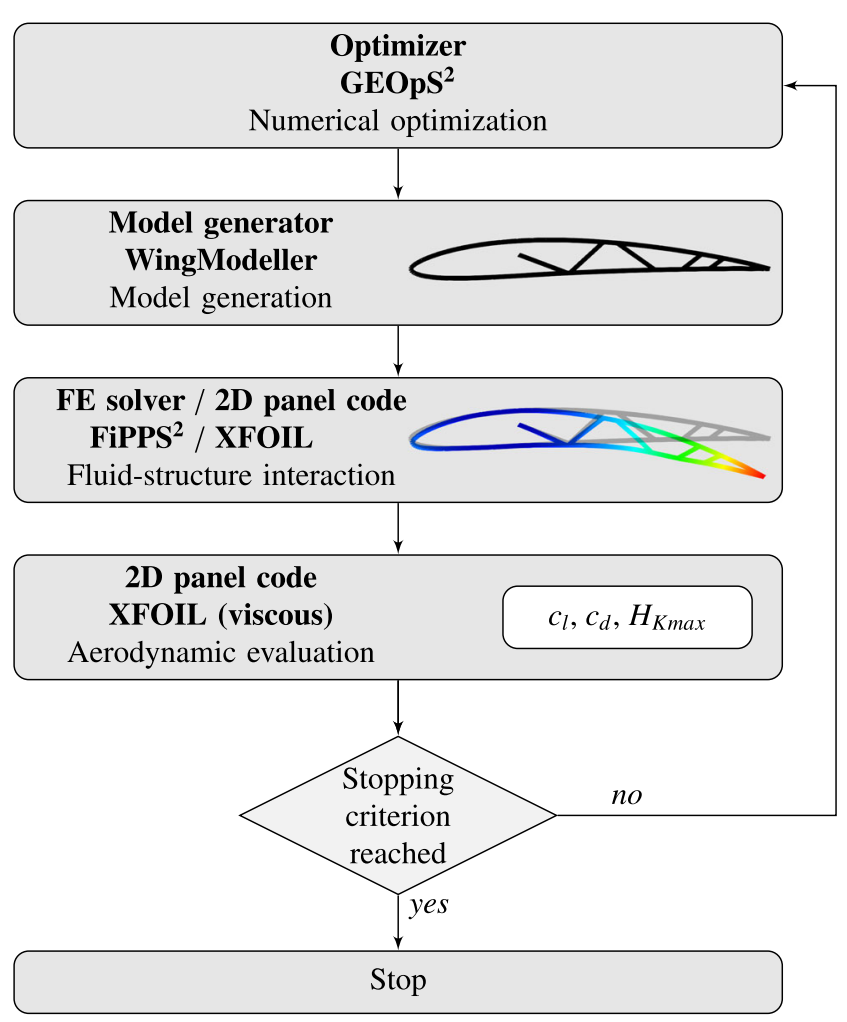

Fig. 11 Outline of the optimization framework
The corresponding penalization parameters are:

$w_{1}= \begin{cases}10^{5} & \text { if the design is infeasible } \\ 1 & \text { otherwise }\end{cases}$

$w_{2}= \begin{cases}\max _{i}\left(\delta A_{i}\right) \cdot 10^{3} & \text { if } \max _{i}\left(\delta A_{i}\right)>0.1 \\ 1 & \text { otherwise }\end{cases}$

with $\delta A_{i}=\left|\frac{A_{i}-A_{1}}{A_{1}}\right|, i=2, \ldots, n_{F C}$,

$w_{3}= \begin{cases}\max _{i}\left(\frac{\Delta \alpha_{i}}{2^{\circ}}\right) \cdot 10^{2} & \text { if } \max _{i}\left(\Delta \alpha_{i}\right)>2^{\circ} \\ 1 & \text { otherwise }\end{cases}$

with $\Delta \alpha_{i}=\left|\alpha\left(c_{l, i}\right)-\alpha\left(c_{l, 1}\right)\right|, i=2, \ldots, n_{F C}$.

As explained in Section 1, no structural constraints are taken into account, as the focus is set on generating layouts of active morphing wing sections that allow an aerodynamically optimized deformation. Hence, structural failure criteria have to be considered when further developing the generated design proposals towards practical realization.

\subsection{Optimization problem}

Taking into account the objectives and constraints mentioned, the resulting multi-objective optimization problem 
Table 2 Applied parameters for evolutionary algorithms

\begin{tabular}{lll}
\hline Algorithm & Description & Value \\
\hline All & Population size & 255 \\
GA & Number of crossover points & 3 \\
& Mutation probability & 0.01 \\
ES & Standard deviation & 0.2 \\
DE & Mutation rate & 0.9 \\
& Number of difference vectors used & 1 \\
& Crossover constant & 0.5 \\
\hline
\end{tabular}

can be stated as

$$
\begin{gathered}
\min _{\mathbf{x}}\left(f_{1}(\mathbf{x}), f_{2}(\mathbf{x}), f_{3}(\mathbf{x}), f_{4}(\mathbf{x})\right) \text { with } \\
f_{1}=\max _{i}\left(c_{d, i}\right) w_{1} w_{2} w_{3} \\
f_{2}=\max _{i}\left(\sigma_{d, i}\right) w_{1} w_{2} w_{3} \\
f_{3}=\max _{i}\left(H_{K \max , i}\right) w_{1} w_{2} w_{3} \\
f_{4}=\mu w_{1} w_{2} w_{3} \\
i=1,2, \ldots, n_{F C}
\end{gathered}
$$

where $\mathbf{x}_{\min } \leq \mathbf{x} \leq \mathbf{x}_{\max }$ contains the optimization variables.

\subsection{Evolutionary optimization}

Equation 13 defines a multi-objective optimization problem consisting of discrete and continuous variables which are listed in Table 1 . With $n_{M M C} \leq 6$ and $n_{F C}=3$, this results in a total of 41 integer and 58 floating point variables. For solving this kind of problem, evolutionary algorithms are very well suited and therefore chosen. Figure 11 shows the outline of the optimization framework.

Basis of the optimization framework is the in-house optimization tool GEOpS ${ }^{2}$ (Kaletta 2006) based on evolutionary algorithms. GEOpS ${ }^{2}$ has been successfully applied to various aerostructural optimization tasks. Examples are the minimal weight design of stiffened fuselage structures by Seeger and Wolf (2011) or the structural optimization of a carbon fiber reinforced polymer flap by Machunze et al. (2016). Efficiency is gained by massive parallelization of the evaluation of individuals.

$\mathrm{GEOpS}^{2}$ uses genetic algorithms (GA), differential evolution (DE), and evolution strategies (ES) as optimization algorithms. The optimization parameters of GA, DE, and ES used for the present problem are given in Table 2. The parameter values have been chosen empirically by manual adaptation in order to achieve reasonable convergence.

The structural model is provided by the in-house model generator WingModeller. For the FE solver and the 2D panel code, the wing is meshed separately. Afterwards, an aerostructural evaluation (described in Section 3.3) is performed for every individual to obtain the shape under actuation and the corresponding aerodynamic loads. Finally, the aerodynamic objectives (described in Section 4.1) of the deformed geometry are determined using XFOIL in viscous mode.

\section{Example}

The aircraft data for specifying the flight conditions of the example presented are listed in Table 3. They are based on the NASA SUGAR High (masses according to 765-095-RD

Table 3 Reference aircraft data

\begin{tabular}{llll}
\hline Description & Variable & Value & Unit \\
\hline Mean aerodynamic chord & $l_{\mu}$ & 2.8012 & $\mathrm{~m}$ \\
Take-off mass & $m_{T O}$ & $62,731.8$ & $\mathrm{~kg}$ \\
Operating empty weight & $m_{O E}$ & $37,058.5$ & $\mathrm{~kg}$ \\
Cruise Mach number & $M a_{c}$ & 0.7 & - \\
Wing area & $S$ & 137.23 & $\mathrm{~m}^{2}$ \\
\hline
\end{tabular}


Table 4 Material properties

\begin{tabular}{llll}
\hline Description & Variable & Value & Unit \\
\hline Young's modulus & $E$ & 73,773 & $\mathrm{MPa}$ \\
Shear modulus & $G$ & 27,734 & $\mathrm{MPa}$ \\
Poisson's ratio & $v$ & 0.33 & - \\
Density & $\rho$ & 2.77 & $\mathrm{~g} / \mathrm{cm}^{3}$ \\
Artificial thermal expansion & $\alpha_{T}$ & 1 & $\mathrm{~K}^{-1}$ \\
\hline
\end{tabular}

Ducted Fan ICAC Constraint) configuration by Bradley et al. (2015).

It is assumed that all structural members are made of the aluminum alloy A12024. The material properties are given in Table 4.

For demonstrating the method, $n_{F C}=3$ flight conditions $i=1,2,3$ are specified in Table 5, where $H$ is the flight altitude and $m_{a c}$ the aircraft mass.

For every flight condition, the lift coefficient is calculated by the vertical force equilibrium of lift and weight

$c_{l}=\frac{2 m_{a c} g}{\rho_{I S A}(H) u_{\infty}^{2} S}$,

where $g=9.81 \mathrm{~m} / \mathrm{s}^{2}$ is the gravitational acceleration.

The flight conditions are based on a simplified mission profile, shown in Fig. 12, in order to define a wide spectrum of lift coefficients. Therefore, the flight conditions considered can be interpreted as extrema in order to reduce drag over the whole mission. Flight conditions 1 and 2 refer to cruise flight with different aircraft masses. For flight condition 1, a minimal mass, slightly higher than the operating empty weight, is assumed. For flight condition 2, a maximum mass, slightly lower than the maximum take-off mass, is considered. The mass reduction can be interpreted as fuel burn during cruise. Flight condition 3 specifies a take-off condition of the aircraft with maximum take-off mass.

The structural model of the wing section has a constant width $w_{a f}=10 \mathrm{~mm}$ in spanwise direction and a chord length of $l=1 \mathrm{~m}$. For demonstration purposes, the design space of the thermal loads is set to $-0.05 \leq T_{j, i} \leq 0.05$. With equation (2) and $\alpha_{T}=1 \mathrm{~K}^{-1}$, this leads to possible actuator strains of $|\varepsilon| \leq 5 \%$. According to Madden et al. (2004), this range corresponds to typical strain limits of SMA actuators.

\subsection{Base airfoil}

The base airfoil shown in Fig. 13 has been designed by applying the aerodynamic objectives described in Section 4.1 to a purely aerodynamic airfoil optimization procedure with the aim to minimize the drag coefficient for $c_{l}=0.4$ of flight condition 1 . As the airfoil optimization for determining the base airfoil is not part of this paper, it is not described in further detail.

The drag polars calculated with XFOIL for $R e=1.31$. $10^{7}$ and $R e=1.44 \cdot 10^{7}$ are shown in Fig. 14. It is clearly visible that the drag coefficient increases significantly at higher lift coefficients.

\subsection{Convergence}

For the optimization, 15,000 generations have been calculated using $256 \mathrm{CPU}$ cores $\left(\right.$ Intel ${ }^{\circledR} \mathrm{Xeon}^{\circledR} \mathrm{E} 5-2680$ v3@2.50 GHz) in parallel, taking approximately $16 \mathrm{~h}$. As a metric for both the convergence and the diversity of the resulting Pareto fronts, the normalized hypervolume indicator, calculated using the method of Fonseca et al. (2006), is shown in Fig. 15.

Table 5 Considered flight conditions (FCs)

\begin{tabular}{lllllllr}
\hline$i$ & $u_{\infty} / \frac{\mathrm{m}}{\mathrm{s}}$ & $H / \mathrm{km}$ & $\rho_{I S A} / \frac{\mathrm{kg}}{\mathrm{m}^{3}}$ & $\eta / \frac{\mathrm{kg}}{\mathrm{m} \cdot \mathrm{s}}$ & $R e /-$ & $M a /-$ & $m_{a c} / \mathrm{kg}$ \\
\hline 1 & 206.55 & 11.5 & 0.34 & $1.48 \cdot 10^{-5}$ & $1.31 \cdot 10^{7}$ & 0.70 & 40,119 \\
2 & 206.55 & 11.5 & 0.34 & $1.48 \cdot 10^{-5}$ & $1.31 \cdot 10^{7}$ & 0.70 & 61,552 \\
3 & 78.11 & 0 & 1.23 & $1.87 \cdot 10^{-5}$ & $1.44 \cdot 10^{7}$ & 0.23 & 62,732 \\
\hline
\end{tabular}




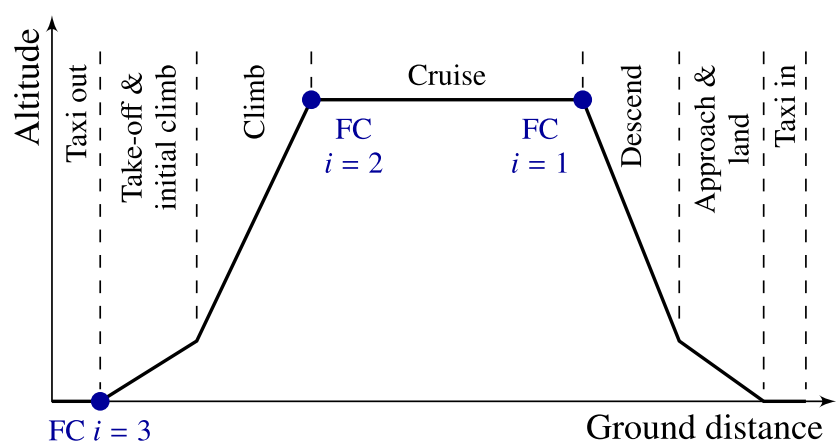

Fig. 12 Simplified mission profile with discrete flight conditions (FCs) specified in Table 5

\subsection{Resulting structures}

For a detailed discussion of the resulting morphing wing section structures, four distinct individuals are selected from the final Pareto front, as shown in Fig. 16. The objective function values of the selected individuals are listed in Table 6.

Individual 1 features a good compromise between a small drag coefficient and a low structural mass. Individual 2 obtains a lower drag coefficient but has a higher structural mass. Individuals 3 and 4 are the individuals with the lowest drag coefficient and lowest structural mass, respectively. Therefore, this selection well represents the bandwidth of resulting structures.

In the following, individual 1 is discussed in further detail. Figure 17 shows the structural details of individual 1. It can be noticed that the internal wall structures have a small thickness compared with the outer wing section contour.

For the first flight condition, the wing section is solely deformed by aerodynamic forces leading to the deformed shape shown in Fig. 18. As in all other figures, the deformed shape is given in true scale. The aerodynamic forces only result in slight deformations due to the compliance of the structure.

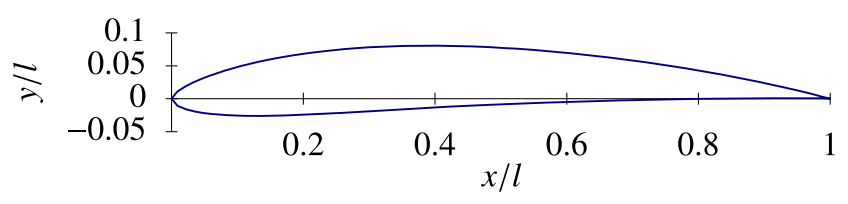

Fig. 13 Contour of the base airfoil



Fig. 14 Drag polars of the base airfoil

The actuation degree for the second flight condition is depicted in Fig. 19. The actuation scheme consists of contraction actuators between $x / l=30 \%$ and $x / l=55 \%$, as well as expansion actuators in the trailing half. Both the internal structure and the wing section skin act partly as active or passive structure. The resulting deformation is shown in Fig. 20. There, the wing trailing half is deflected, leading to an increased camber.

Finally, for the third flight condition, the actuation scheme is adjusted by the optimizer (see Fig. 21). The actuation degree of the contraction actuators between $x / l=$ $30 \%$ and $x / l=55 \%$ is increased. The actuators in the trailing edge region change from expansion to contraction. This creates a second deflection point and further increases the airfoil camber, as visible in Fig. 22.



Fig. 15 Development of the normalized hypervolume indicator over the optimization progress 


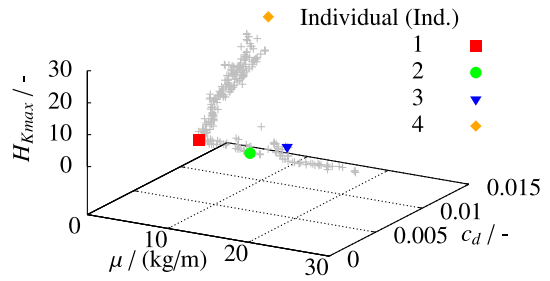

(a) Axes $\mu-c_{d}-H_{K \max }$



(d) Axes $\mu-c_{d}-\sigma_{d}$

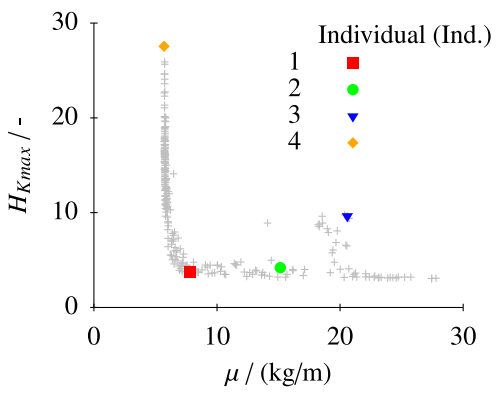

(b) Axes $\mu-H_{K \max }$

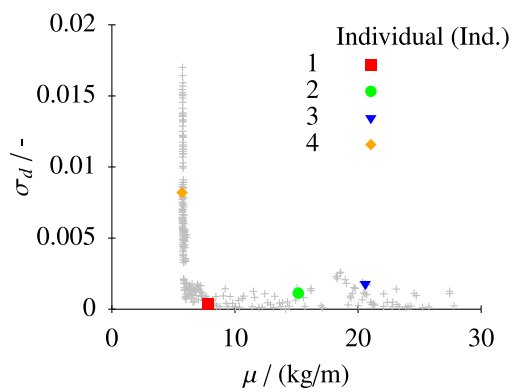

(e) Axes $\mu-\sigma_{d}$

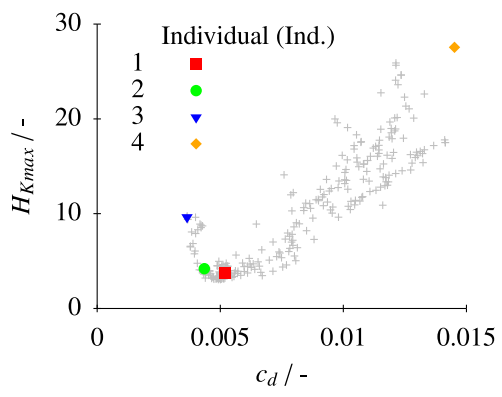

(c) Axes $c_{d}-H_{K \max }$

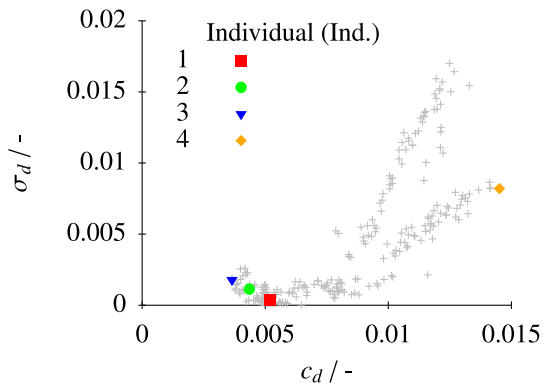

(f) Axes $c_{d}-\sigma_{d}$

Fig. 16 Resulting Pareto front with selected individuals, projected in three-dimensional views (a), (d) and two-dimensional views (b), (c), (e), (f)

Table 6 Objective function values of selected individuals

\begin{tabular}{lllll}
\hline Individual & $\mu /(\mathrm{kg} / \mathrm{m})$ & $c_{d} /-$ & $\sigma_{d} /-$ & $H_{K m a x} /-$ \\
\hline 1 & 7.84552 & 0.00519 & 0.00037 & 3.77533 \\
2 & 15.1462 & 0.00435 & 0.00113 & 4.19502 \\
3 & 20.5862 & 0.00365 & 0.00178 & 9.65291 \\
4 & 5.69445 & 0.01451 & 0.00820 & 27.5551 \\
\hline
\end{tabular}

Fig. 17 Individual 1:

Normalized structural thickness



Normalized thickness $t / /$
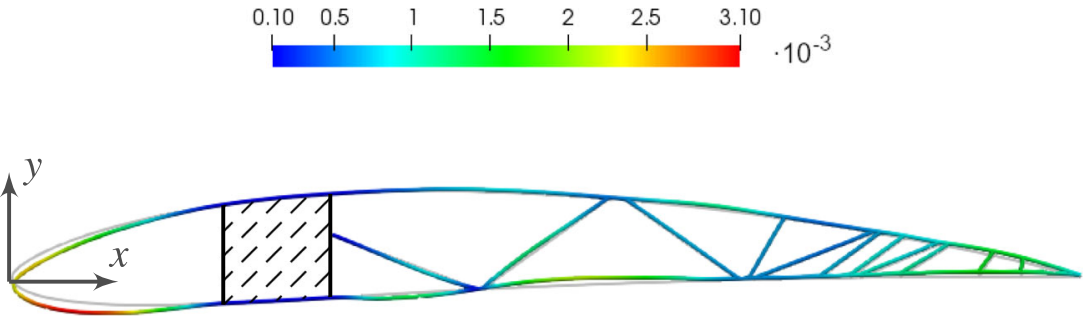

Normalized displacement vector sum $|\mathbf{u}| / \mid$

$\begin{array}{lllllll}0.00 & 2 & 4 & 6 & 8 & 9.39\end{array}$

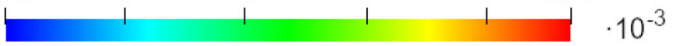


Fig. 19 Individual 1: Actuation degrees $T_{j, 2}$ for flight condition $i=2$

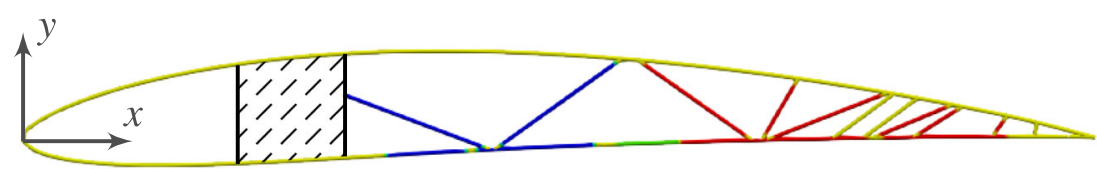

Details of individuals 2 to 4 are given by Figs. 25, 26, and 27 in the Appendix. In the following, they are discussed only in brief, to show the bandwidth of the results.

Compared with individual 1 , the internal structure of individual 2 consists of less structural members but has an increased thickness, leading to a significantly higher mass. The overall deformation behavior is similar to that of individual 1 for all three flight conditions.

Individual 3 is selected from the Pareto front as the solution having the smallest drag coefficient $c_{d}$. It is noticeable that this individual does not contain any internal structure and consists only of an outer skin with a relatively large thickness. This leads to an increased mass compared with the other individuals discussed.

Individual 4 represents the solution with the lowest specific mass $\mu$ in the Pareto front. This is achieved by comparably low thickness values of the walls, leading to a high compliance under aerodynamic loads. This results in high drag coefficients $c_{d} \geq 0.01451$. Thus, this individual does not improve the aerodynamic efficiency compared with the undeformed wing section.

\subsection{Aerodynamic performance}

For further evaluation of the aerodynamic performance, the drag polars of the morphing wing sections are shown in Fig. 23 compared with the drag polar of the undeformed base airfoil and the drag polar of the NACA 2412 airfoil.
Actuation degree, flight condition $2 /$ -

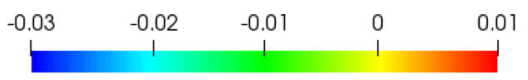

Additionally, the drag polar of the NACA 2412 airfoil with a conventional trailing edge flap over the last $20 \%$ of the chord length, deflected $10^{\circ}$ downwards (see Fig. 24), is shown for comparison. As individual 4 obtains the lowest structural mass on the expense of highly increased drag coefficients, it is not shown here. The polars shown have been generated by interpolating linearly the actuation degrees $T_{j, i}$ for the three flight conditions. This also applies to the lift coefficient $c_{l, i}$, the Mach number $M a_{i}$ and the Reynolds number $R e_{i}$. An individual aerostructural evaluation has been performed for every interpolated lift coefficient.

As stated in Section 3.3, the applied aerodynamic solver XFOIL is well suited for calculating aerodynamic characteristics of common airfoil shapes. Except for individual 4 , the contours of the actuated wing sections do not differ significantly from common airfoils. However, individual 4 shows a non-smooth contour under actuation (see Fig. 27) but obtains high drag coefficients, accordingly. In consequence, it can be concluded that the use of XFOIL is feasible in the present method for evaluating the aerodynamic performance with low computational effort.

As Fig. 23 shows, the minimal drag coefficient for each wing section is almost constant over the varying flight conditions. This is in accordance with the aimed targets for morphing wing sections, as defined in Section 2. Thus, it is possible to morph the wing sections continuously when changing between distinct flight conditions in order
Fig. 20 Individual 1:

Normalized displacement vector sum for flight condition $i=2$

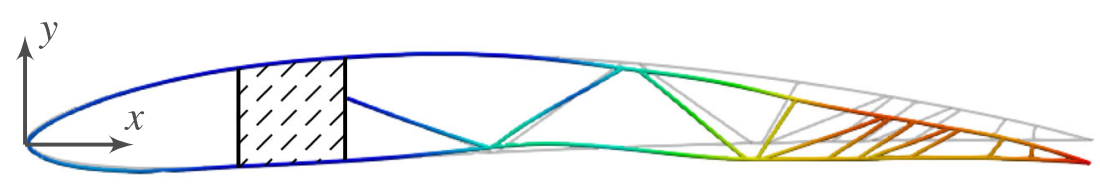

Normalized displacement vector sum $|\mathbf{u}| / /$

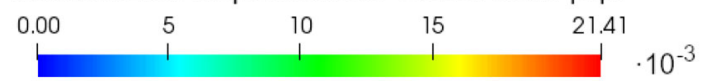


Fig. 21 Individual 1: Actuation degrees $T_{j, 3}$ for flight condition $i=3$

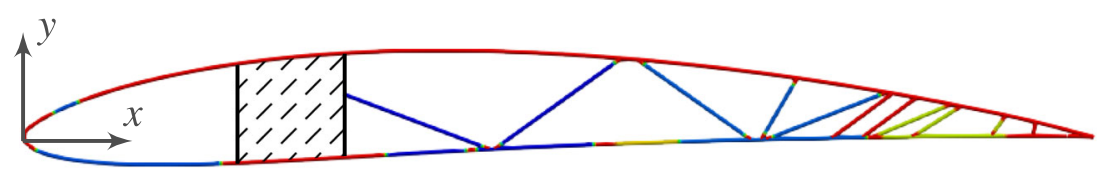

to maintain a minimized drag coefficient. This can be achieved even though only three discrete flight conditions are considered in the optimization run.

Taking a closer look at the polars, it can be seen that the drag coefficient of individual 1 for flight condition 1 is slightly increased compared with the undeformed wing section. For flight condition 2, the deformed wing section obtains a slightly lower drag coefficient than the undeformed airfoil; for flight condition 3 , the drag coefficient is significantly lower than the drag coefficient of the undeformed airfoil. Compared with the polar of the NACA 2412 airfoil, the drag coefficients are lower for every flight condition considered here. This also applies to the comparison with the polar of the NACA 2412 airfoil with a trailing edge flap. In this case, the drag benefit of the morphing sections is even higher. Furthermore, individuals 2 and 3 have significantly reduced drag coefficients compared with the polar of the undeformed base airfoil. Hence, they show a better aerodynamic performance as individual 1 , with the drawback of higher structural masses.

\subsection{Summary of results}

The investigated examples prove that the current optimization approach enables to find active morphing wing section structures that minimize the aerodynamic drag for each of the three given flight conditions compared with the undeformed base airfoil. The resulting wing sections further allow the linear interpolation of the actuation
Actuation degree, flight condition 3 / -

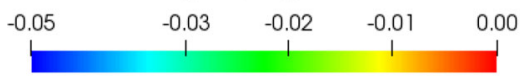

degree in between the flight conditions without significant drag increase. Therefore, a low discrete number of flight conditions are sufficient as optimization targets in order to generate continuously morphing sections.

Sections with a low structural mass have more internal structural members than those with a higher structural mass. In contrast, sections with a large number of internal structural members feature higher drag coefficients as their higher flexibility leads to undesired deformations of the outer contour. Thus, the structural mass $m$ of the active morphing wing section and the resulting drag coefficient $c_{d}$ are opposite targets in the presented approach.

Although, the parameterization of the wing section structure and the free actuator placement allow highly unrestricted deformations of the actuated sections, aerodynamically sensible results mainly show an increase in the airfoil camber resembling structurally integrated trailing edge flaps.

\section{Conclusion}

This paper presents an approach for the design optimization of the internal structure of an active morphing wing section including linear contraction and expansion actuators. Neither the target shape of the wing section nor the internal structural layout and the actuation scheme are defined a priori. Both the structure and the target shape are optimized concurrently using a fluid-structure interaction approach of a linear in-house finite element solver and the
Fig. 22 Individual 1:

Normalized displacement vector sum for flight condition $i=3$

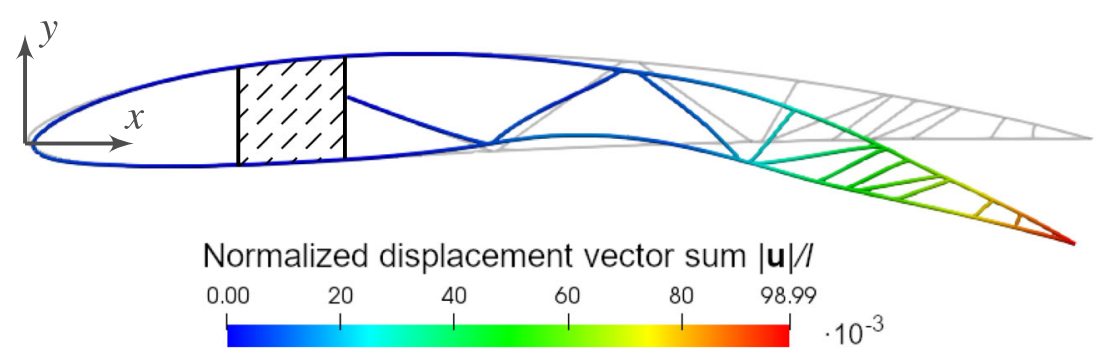




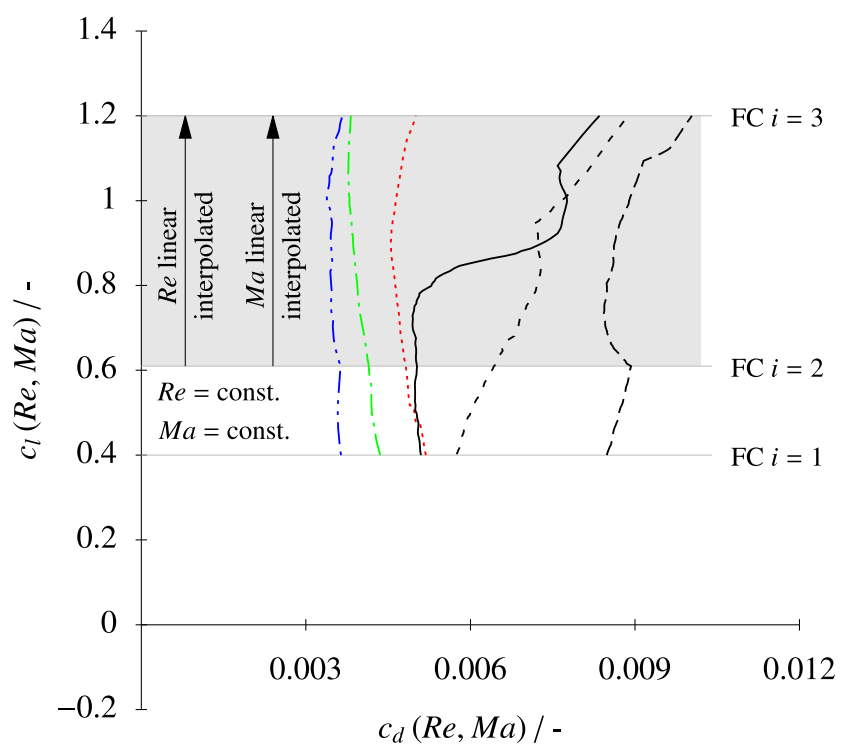

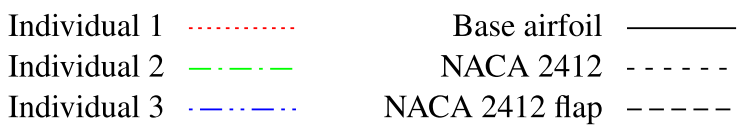

Fig. 23 Drag polars of the selected morphing wing sections with flight conditions (FCs) specified in Table 5

viscous-inviscid two-dimensional panel code XFOIL. The optimization is performed using evolutionary algorithms. Thereby, their suitability for parallel computing has been exploited. Lindenmayer cellular systems are applied as an efficient parameterization method of the internal structure.

An advantage of this approach is that it requires only a very small number of input data. The user just has to specify the desired target flight conditions, the base airfoil, the range of actuation degrees, and the placement of a rigid spar area. All input requirements are physically based and describe the boundary conditions of an engineering problem.

It could be shown that the presented approach is suitable to optimize active morphing wing sections fulfilling the requested aerodynamic objectives and leading to reduced drag coefficients over varying flight conditions. Hence,

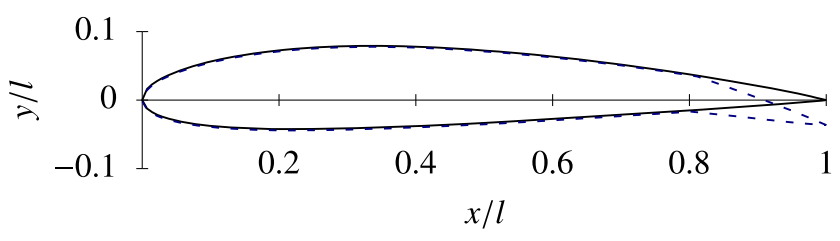

\section{NACA $2412-\quad$ NACA 2412 flap $\ldots \ldots$}

Fig. 24 Contour of the NACA 2412 airfoil with and without trailing edge flap used for the comparison of drag polars in Fig. 23 in a highly automated process, design proposals for morphing wing sections can be generated in reasonable time. Detailed discussions of the Pareto front, resulting from the multi-objective optimization, further allow the deduction of basic knowledge relevant to the design of morphing wing concepts. Furthermore, the Pareto front offers a wide range of structural solutions from which an engineer can choose a suitable one by weighting the objectives individually.

Further research will be done in order to investigate alternative parameterization methods for the internal structure, to reduce the number of objectives of the optimization problem, and to integrate suitable structural failure constraints. Another focus is set on the replacement of XFOIL with aerodynamic solvers of higher order and the integration of an aerodynamic convergence criterion in the fluid-structure interaction. In addition, a simultaneous optimization of the base airfoil will be included, with the aim of eliminating the need for a predefinition.

Acknowledgements Calculations have been performed on the Bull HPC-cluster of the Zentrum für Informationsdienste und Hochleistungsrechnen $(\mathrm{ZIH})$ of the Technische Universität Dresden.

Funding information Open Access funding provided by Projekt DEAL. This research was funded by the German Federal Ministry for Economic Affairs and Energy under grant number 20E1509B. The responsibility for the content of this paper is with its authors. The financial support is gratefully acknowledged.

\section{Compliance with ethical standards}

Conflict of interest The authors declare that they have no conflict of interest.

Replication of results Results can be replicated by adopting the presented optimization method with limitations due to the heuristic nature of the applied evolutionary algorithms. As the optimization method is implemented with an extensive use of in-house software not being publicly available, no supplementary computer code is supplied.

Open Access This article is licensed under a Creative Commons Attribution 4.0 International License, which permits use, sharing, adaptation, distribution and reproduction in any medium or format, as long as you give appropriate credit to the original author(s) and the source, provide a link to the Creative Commons licence, and indicate if changes were made. The images or other third party material in this article are included in the article's Creative Commons licence, unless indicated otherwise in a credit line to the material. If material is not included in the article's Creative Commons licence and your intended use is not permitted by statutory regulation or exceeds the permitted use, you will need to obtain permission directly from the copyright holder. To view a copy of this licence, visit http://creativecommons. org/licenses/by/4.0/. 


\section{Appendix}
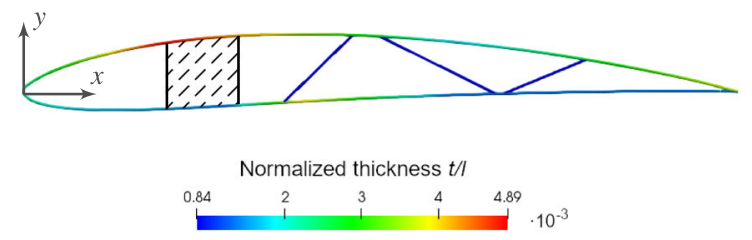

(a) Normalized structural thickness

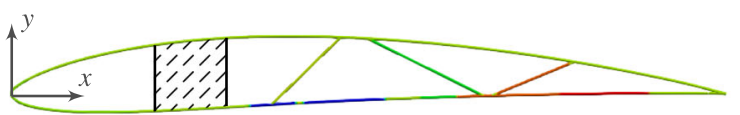

Actuation degree, flight condition $2 /$ -

\begin{tabular}{cccccc}
-0.03 & -0.02 & -0.01 & 0 & 0.01 & 0.02 \\
\hline
\end{tabular}

(c) Actuation degrees $T_{j, 2}$ for flight condition $i=2$

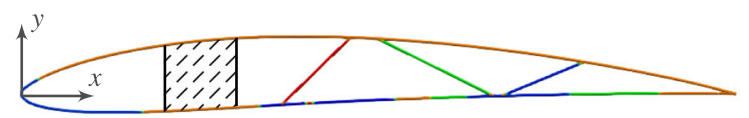

Actuation degree, flight condition $3 /$ -
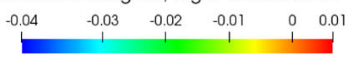

(e) Actuation degrees $T_{j, 3}$ for flight condition $i=3$

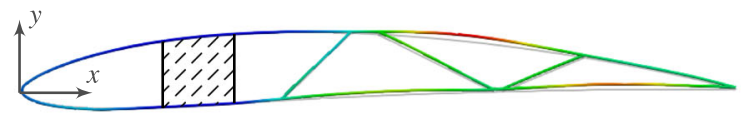

Normalized displacement vector sum $|\mathbf{u}| / \mid$

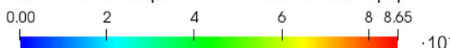

(b) Normalized displacement vector sum, flight condition $i=1$



Normalized displacement vector sum $|\mathbf{u}| / \mid$

$\begin{array}{lllllllll}0.00 & 2 & 4 & 6 & 8 & 10 & 12 & 14 & 15.62\end{array}$

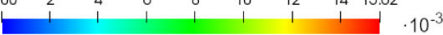

(d) Normalized displacement vector sum, flight condition $i=2$

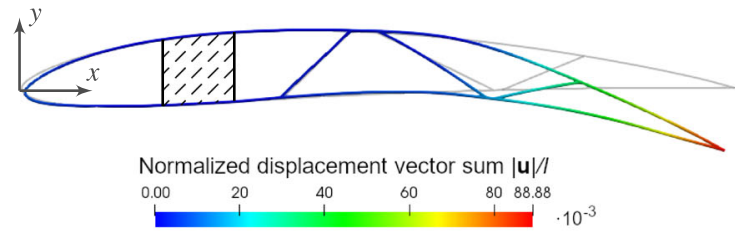

(f) Normalized displacement vector sum, flight condition $i=3$

Fig. 25 Individual 2: Normalized structural thickness (a), actuation degrees for flight conditions 2 (c) and 3 (e), and deformation behavior for flight conditions 1 (b), 2 (d) and 3 (f)


(a) Normalized structural thickness

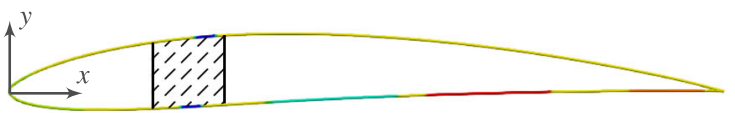

Actuation degree, flight condition $2 /$ -

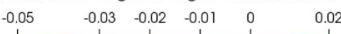

$\begin{array}{lllll}1 & 1 & 1 & 1\end{array}$

(c) Actuation degrees $T_{j, 2}$ for flight condition $i=2$

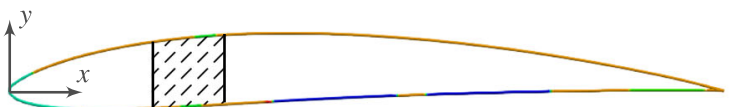

Actuation degree, flight condition $3 /$ -

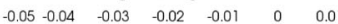

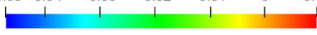

(e) Actuation degrees $T_{j, 3}$ for flight condition $i=3$



Normalized displacement vector sum $|\mathbf{u}| / \mid$

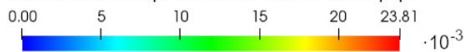

(b) Normalized displacement vector sum, flight condition $i=1$



Normalized displacement vector sum $|\mathbf{u}| / \mid$

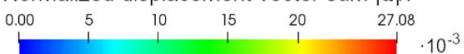

(d) Normalized displacement vector sum, flight condition $i=2$



(f) Normalized displacement vector sum, flight condition $i=3$

Fig. 26 Individual 3: Normalized structural thickness (a), actuation degrees for flight conditions 2 (c) and 3 (e), and deformation behavior for flight conditions 1 (b), 2 (d) and 3 (f) 


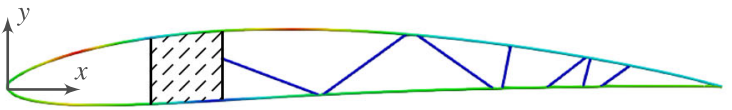

Normalized thickness $t / 1$

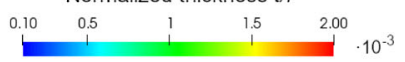

(a) Normalized structural thickness



Actuation degree, flight condition 2 / -

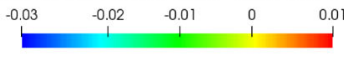

(c) Actuation degrees $T_{j, 2}$ for flight condition $i=2$

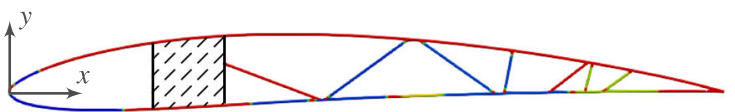

Actuation degree, flight condition $3 /$ -

\begin{tabular}{ccccc}
-0.04 & -0.03 & -0.02 & -0.01 & 0.00 \\
\hline
\end{tabular}

(e) Actuation degrees $T_{j, 3}$ for flight condition $i=3$

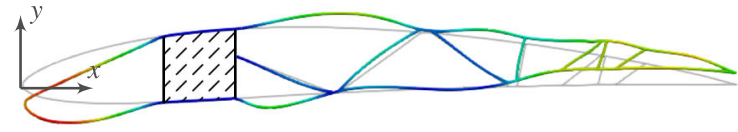

Normalized displacement vector sum $|\mathbf{u}| / \mid$

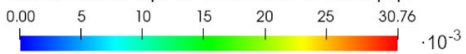

(b) Normalized displacement vector sum, flight condition $i=1$

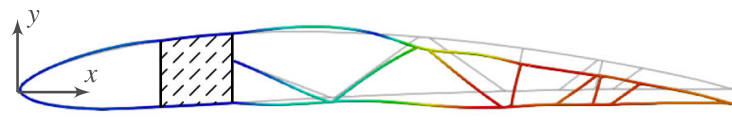

Normalized displacement vector sum $|\mathbf{u}| / \mid$

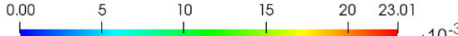

(d) Normalized displacement vector sum, flight condition $i=2$

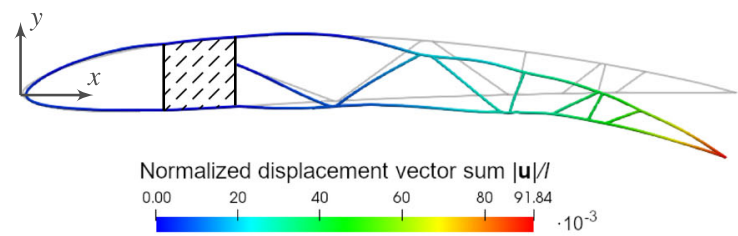

(f) Normalized displacement vector sum, flight condition $i=3$

Fig. 27 Individual 4: Normalized structural thickness (a), actuation degrees for flight conditions 2 (c) and 3 (e), and deformation behavior for flight conditions 1 (b), 2 (d) and 3 (f)

\section{References}

Baker D, Friswell MI (2009) Determinate structures for wing camber control. Smart Mater Struct 18(3):035014. https://doi.org/10.1088/ 0964-1726/18/3/035014

Barbarino S, Bilgen O, Ajaj RM, Friswell MI, Inman DJ (2011) A review of morphing aircraft. J Intell Material Syst Struct 22(9):823-877. https://doi.org/10.1177/1045389x11414084

Bradley MK, Droney CK, Allen TJ (2015) Subsonic ultra green aircraft research: phase II - volume I - truss braced wing design exploration. Tech. Rep. NASA/CR-2015-218704/Volume I, National Aeronautics and Space Administration, Hampton, https:// ntrs.nasa.gov/archive/nasa/casi.ntrs.nasa.gov/20150017036.pdf

De Gaspari A, Ricci S (2011) A two-level approach for the optimal design of morphing wings based on compliant structures. J Intell Material Syst Struct 22(10):1091-1111. https://doi.org/10.1177/1045389x11409081

Deaton JD, Grandhi RV (2014) A survey of structural and multidisciplinary continuum topology optimization: post 2000. Struct Multidiscipl Optim 49(1):1-38. https://doi.org/10.1007/s00158-0130956-Z

Drela M (1989) XFOIL: an analysis and design system for low reynolds number airfoils. In: Mueller TJ (ed) Low Reynolds Number Aerodynamics. Springer, Berlin, pp 1-12. https://doi.org/10.1007/978-3-642-84010-4_1

Drela M (1998) Pros \& cons of airfoil optimization. In: Caughey DA, Hafez MM (eds) Frontiers of Computational Fluid Dynamics 1998. World Scientific, Singapore, pp 363-381. https://doi.org/10.1142/9789812815774_0019
European Commission (2011) Flightpath 2050: Europe's vision for aviation. Tech. rep., Publications Office of the European Union, Luxembourg, https://doi.org/10.2777/50266, https://ec.europa.eu/ transport/sites/transport/files/modes/air/doc/flightpath2050.pdf

Fincham JHS, Friswell MI (2015) Aerodynamic optimisation of a camber morphing aerofoil. Aerosp Sci Technol 43:245-255. https://doi.org/10.1016/j.ast.2015.02.023

Fonseca CM, Paquete L, López-Ibáñez M (2006) An improved dimension-sweep algorithm for the hypervolume indicator. In: 2006 IEEE Congress on Evolutionary Computation, Vancouver, pp 1157-1163. https://doi.org/10.1109/CEC.2006.1688440

Friswell MI, Baker D, Herencia JE, Mattioni F, Weaver PM (2006) Compliant structures for morphing aircraft. In: Proceedings of ICAST2006, Taipei, pp 17-24

Fusi F, Congedo PM, Guardone A, Quaranta G (2018) Shape optimization under uncertainty of morphing airfoils. Acta Mech 229(3):1229-1250. https://doi.org/10.1007/s00707-017-2049-3

Guo X, Zhang W, Zhong W (2014) Doing topology optimization explicitly and geometrically-a new moving morphable components based framework. J Appl Mech 81(8):081009. https://doi.org/10.1115/1.4027609

Herbert-Acero JF, Probst O, Rivera-Solorio CI, Castillo-Villar KK, Méndez-Díaz S (2015) An extended assessment of fluid flow models for the prediction of two-dimensional steadystate airfoil aerodynamics. Math Probl Eng 2015:854308. https://doi.org/10.1155/2015/854308

Kaletta P (2006) Ein Beitrag zur Effizienzsteigerung Evolutionärer Algorithmen zur optimalen Auslegung von Faserverbundstrukturen im Flugzeugbau. Dissertation, Technische Universität Dresden 
Li D, Zhao S, Da Ronch A, Xiang J, Drofelnik J, Li Y, Zhang L, Wu Y, Kintscher M, Monner HP, Rudenko A, Guo S, Yin W, Kirn J, Storm S, De Breuker R (2018) A review of modelling and analysis of morphing wings. Prog Aerosp Sci 100:46-62. https://doi.org/10.1016/j.paerosci.2018.06.002

Machunze W, Gessler A, Fabel T, Horst P, Rädel M, Wolf K, Ulbricht A, Münter S, Hufenbach W (2016) Active flow control system integration into a CFRP, flap. CEAS Aeronaut J 7(1):69-81. https://doi.org/10.1007/s13272-015-0171-2

Madden JDW, Vandesteeg NA, Anquetil PA, Madden PGA, Takshi A, Pytel RZ, Lafontaine SR, Wieringa PA, Hunter IW (2004) Artificial muscle technology: physical principles and naval prospects. IEEE J Ocean Eng 29(3):706-728. https://doi.org/10.1109/JOE.2004.833135

Maute K, Reich GW (2006) Integrated multidisciplinary topology optimization approach to adaptive wing design. J Aircr 43(1):253263. https://doi.org/10.2514/1.12802

Molinari G, Arrieta AF, Ermanni P (2014) Aero-structural optimization of three-dimensional adaptive wings with embedded smart actuators. AIAA J 52(9):1940-1951. https://doi.org/10.2514/1. J052715

Namgoong H, Crossley WA, Lyrintzis AS (2012) Morphing airfoil design for minimum drag and actuation energy including aerodynamic work. J Aircr 49(4):981-990. https://doi.org/10.2514/1. C031395

Pedro HTC, Kobayashi MH (2011) On a cellular division method for topology optimization. Int J Numer Meth Eng 88(11):1175-1197. https://doi.org/10.1002/nme.3218
Prusinkiewicz P, Lindenmayer A (1990) The algorithmic beauty of plants, the virtual laboratory, vol 1. Springer, New York. https://doi.org/10.1007/978-1-4613-8476-2

Secanell M, Suleman A, Gamboa P (2006) Design of a morphing airfoil using aerodynamic shape optimization. AIAA J 44(7):1550 1562. https://doi.org/10.2514/1.18109

Seeger J, Wolf K (2011) Multi-objective design of complex aircraft structures using evolutionary algorithms. P I Mech Eng G-J Aerosp Eng 225(10):1153-1164. https://doi.org/10.1177/ 0954410011411384

Seeger J (2012) Ein Beitrag zur numerischen Strukturauslegung aktiver Rotorblätter unter Berücksichtigung der Wechselwirkung von Strömung und Struktur. Dissertation. Technische Universität Dresden

Strelec JK, Lagoudas DC, Khan MA, Yen J (2003) Design and implementation of a shape memory alloy actuated reconfigurable airfoil. J Intell Material Syst Struct 14(4-5):257-273. https://doi.org/10.1177/1045389x03034687

Vasista S, Tong L, Wong KC (2012) Realization of morphing wings: a multidisciplinary challenge. J Aircr 49(1):11-28. https://doi.org/10.2514/1.C031060

Woods BKS, Friswell MI (2016) Multi-objective geometry optimization of the fish bone active camber morphing airfoil. J Intell Material Syst Struct 27(6):808-819. https://doi.org/10.1177/ 1045389x15604231

Publisher's note Springer Nature remains neutral with regard to jurisdictional claims in published maps and institutional affiliations. 